\title{
A Note on Total and Paired Domination of Cartesian Product Graphs
}

\author{
K. Choudhary \\ Department of Mathematics and Statistics \\ Indian Institute of Technology Kanpur \\ Kanpur, India \\ keerti.india@gmail.com
}

\author{
S. Margulies \\ Department of Mathematics \\ Pennsylvania State University \\ State College, PA, U.S.A. \\ margulies@math.psu.edu
}

\author{
I. V. Hicks \\ Department of Computational and Applied Mathematics \\ Rice University \\ Houston, TX, U.S.A. \\ ivhicks@rice.edu
}

Submitted: Sep 9, 2011; Accepted: Jul 27, 2013; Published: Aug 23, 2013

Mathematics Subject Classifications: 05C69

\begin{abstract}
A dominating set $D$ for a graph $G$ is a subset of $V(G)$ such that any vertex not in $D$ has at least one neighbor in $D$. The domination number $\gamma(G)$ is the size of a minimum dominating set in $G$. Vizing's conjecture from 1968 states that for the Cartesian product of graphs $G$ and $H, \gamma(G) \gamma(H) \leqslant \gamma(G \square H)$, and Clark and Suen (2000) proved that $\gamma(G) \gamma(H) \leqslant 2 \gamma(G \square H)$. In this paper, we modify the approach of Clark and Suen to prove similar bounds for total and paired domination in the general case of the $n$-Cartesian product graph $A_{1} \square \cdots \square A_{n}$. As a by-product of these results, improvements to known total and paired domination inequalities follow as natural corollaries for the standard $G \square H$.
\end{abstract}

\section{Introduction}

We consider simple undirected graphs $G=(V, E)$ with vertex set $V$ and edge set $E$. The open neighborhood of a vertex $v \in V(G)$ is denoted by $N_{G}(v)$, and closed neighborhood by $N_{G}[v]$. A dominating set $D$ of a graph $G$ is a subset of $V(G)$ such that for all $v$, $N_{G}[v] \cap D \neq \emptyset$. A $\gamma$-set of $G$ is a minimum dominating set for $G$, and its size is denoted $\gamma(G)$. A total dominating set $D$ of a graph $G$ is a subset of $V(G)$ such that for all $v$, $N_{G}(v) \cap D \neq \emptyset$. A $\gamma_{t}$-set of $G$ is a minimum total dominating set for $G$, and its size is 
denoted $\gamma_{t}(G)$. A paired dominating set $D$ for a graph $G$ is a dominating set such that the subgraph of $G$ induced by $D$ (denoted $G[D]$ ) has a perfect matching. A $\gamma_{p r}$-set of $G$ is a minimum paired dominating set for $G$, and its size is denoted $\gamma_{p r}(G)$. In general, for a graph containing no isolated vertices, $\gamma(G) \leqslant \gamma_{t}(G) \leqslant \gamma_{p r}(G)$.

The Cartesian product graph, denoted $G \square H$, is the graph with vertex set $V(G) \times$ $V(H)$, where vertices $g h$ and $g^{\prime} h^{\prime}$ are adjacent whenever $g=g^{\prime}$ and $\left(h, h^{\prime}\right) \in E(H)$, or $h=h^{\prime}$ and $\left(g, g^{\prime}\right) \in E(G)$. Just as the Cartesian product of graphs $G$ and $H$ is denoted $G \square H$, the $n$-product of graphs $A_{1}, A_{2}, \ldots, A_{n}$ is denoted as $A_{1} \square A_{2} \square \cdots \square A_{n}$, and has vertex set $V\left(A_{1}\right) \times V\left(A_{2}\right) \times \cdots \times V\left(A_{n}\right)$, where vertices $u_{1} \cdots u_{n}$ and $v_{1} \cdots v_{n}$ are adjacent if and only if for some $i,\left(u_{i}, v_{i}\right) \in E\left(A_{i}\right)$, and $u_{j}=v_{j}$ for all other indices $j \neq i$.

Vizing's conjecture from 1968 states that $\gamma(G) \gamma(H) \leqslant \gamma(G \square H)$. For a thorough review of the activity on this famous open problem, see [1] and references therein. In 2000, Clark and Suen [3] proved that $\gamma(G) \gamma(H) \leqslant 2 \gamma(G \square H)$ by a sophisticated doublecounting argument which involved projecting a $\gamma$-set of the product graph $G \square H$ down onto the graph $H$. In this paper, we slightly modify the Clark and Suen double-counting approach and instead project subsets of $G \square H$ down onto both graphs $G$ and $H$, which allows us to prove several theorems/corollaries relating to total and paired domination. In this section, we state the results, and in Section 2, we prove the results.

Theorem 1. Given graphs $A_{1}, A_{2}, \ldots, A_{n}$ containing no isolated vertices,

$$
\gamma\left(A_{1}\right) \prod_{i=2}^{n} \gamma_{t}\left(A_{i}\right) \leqslant n \gamma\left(A_{1} \square A_{2} \square \cdots \square A_{n}\right) .
$$

In 2008, Ho [4] proved $\gamma_{t}(G) \gamma_{t}(H) \leqslant 2 \gamma_{t}(G \square H)$, an inequality for total domination precisely analogous to the Clark and Suen inequality for domination. In this paper, we extend this result to the $n$-product case, and then Ho's inequality becomes a special case of a more general result.

Theorem 2. Given graphs $A_{1}, A_{2}, \ldots, A_{n}$ containing no isolated vertices,

$$
\prod_{i=1}^{n} \gamma_{t}\left(A_{i}\right) \leqslant n \gamma_{t}\left(A_{1} \square A_{2} \square \cdots \square A_{n}\right) .
$$

In 2007, Brešar, Henning and Rall [2] proved that $\gamma_{p r}(G) \gamma_{p r}(H) \leqslant 8 \gamma_{p r}(G \square H)$, and in 2010, Hou and Jiang [5] proved that $\gamma_{p r}(G) \gamma_{p r}(H) \leqslant 7 \gamma_{p r}(G \square H)$. We extend these results to the $n$-product case, and attain an improvement to these inequalities as a corollary:

Theorem 3. Given graphs $A_{1}, \ldots, A_{n}$ containing no isolated vertices,

$$
\prod_{i=1}^{n} \gamma_{p r}\left(A_{i}\right) \leqslant 2^{n-1}(2 n-1) \gamma_{p r}\left(A_{1} \square \cdots \square A_{n}\right) .
$$

Corollary. Given graphs $G$ and $H$ containing no isolated vertices,

$$
\gamma_{p r}(G) \gamma_{p r}(H) \leqslant 6 \gamma_{p r}(G \square H)
$$




\section{Main Results}

We begin by introducing some notation which will be utilized throughout the proofs in this section. Given $S \subseteq V\left(A_{1} \square \cdots \square A_{n}\right)$, the projection of $S$ onto graph $A_{i}$ is defined as

$$
\Phi_{A_{i}}(S)=\left\{a \in V\left(A_{i}\right) \mid \exists u_{1} \cdots u_{n} \in S \text { with } a=u_{i}\right\}
$$

We partition the set of edges $E\left(A_{1} \square \cdots \square A_{n}\right)$ into $n$ sets. Thus, we define $E_{i}$ to be

$$
E_{i}=\left\{\left(u_{1} \cdots u_{n}, v_{1} \cdots v_{n}\right) \mid\left(u_{i}, v_{i}\right) \in E\left(A_{i}\right), \text { and } u_{j}=v_{j} \text {, for all other indices } j \neq i\right\} .
$$

An edge $e \in E_{i}$ is said to be an $E_{i}$-edge. For $u \in V\left(A_{1} \square \cdots \square A_{n}\right)$, the $i$-neighborhood of $u$ is defined as follows:

$$
N_{\square A_{i}}(u)=\left\{v \in V\left(A_{1} \square \cdots \square A_{n}\right) \mid v \text { and } u \text { are connected by } E_{i} \text {-edge }\right\} .
$$

Finally, we present a proposition utilized throughout our proofs. Although the more general $n$-dimensional case stated in Prop. 2 is the proposition referenced within the proofs, we begin by separately stating the 2-dimensional case to clarify the overall idea.

Proposition 1. Let $M$ be a matrix containing only $0 / 1$ entries. Then at least one of the following two statements are true:

(a) each column contains a 1,

(b) each row contains a 0 .

Prop. 1 refers only to binary matrices, or matrices containing only $0 / 1$ entries. Prop. 2 refers to $n$-ary matrices, or (in this case) matrices containing only entries in $\{1, \ldots, n\}$. Furthermore, Prop. 1 refers only to two-dimensional matrices, or $d_{1} \times d_{2}$ matrices $M$. Prop. 2 refers to $n$-dimensional matrices, or $d_{1} \times d_{2} \times \cdots \times d_{n}$ matrices $M$.

Definition 1. Let $M$ be a $d_{1} \times d_{2} \times \cdots \times d_{n}$, n-ary matrix. Then $M$ is a $j$-matrix if there exists a $j \in\{1, \ldots, n\}$ (not necessarily unique), such that each of the $\overline{d_{1} \times \cdots \times} d_{j-1} \times$ $1 \times d_{j+1} \times \cdots \times d_{n}$ submatrices of $M$ contains an entry with value $j$.

Proposition 2. Every $d_{1} \times d_{2} \times \cdots \times d_{n}$, n-ary matrix $M$ is a $j$-matrix for some $j \in$ $\{1, \ldots, n\}$ (not necessarily unique).

Note that, given any $d_{1} \times d_{2} \times \cdots \times d_{n}$ matrix, there are $d_{j}$ submatrices of the form $d_{1} \times \cdots \times d_{j-1} \times 1 \times d_{j+1} \times \cdots \times d_{n}$. Following standard MATLAB notation, we will denote such a submatrix as $M\left[:, \ldots,:, i_{j},:, \ldots,:\right]$ with $1 \leqslant i_{j} \leqslant d_{j}$.

Example 1. Here we see a $4 \times 3 \times 3$, 3-ary (the entries are contained in the set $\{1,2,3\}$ ) matrix $M$. Using the notation specified above, $M[2,:,:]$ is denoted by the gray shaded region and $M[:, 1,:]$ is denoted by yellow shaded region. $M$ is both 1-matrix and 2-matrix, but not a 3-matrix. 


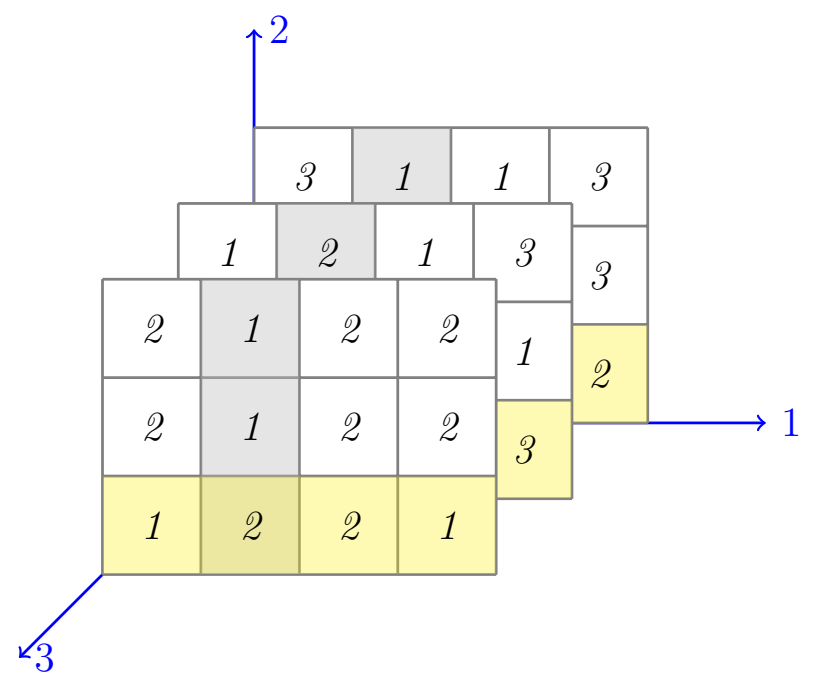

Proof. For a pigeon-hole principle style proof, let $M$ be a $d_{1} \times d_{2} \times \cdots \times d_{n} n$-ary matrix which is not a $j$-matrix for $1 \leqslant j \leqslant n-1$. We will show that $M$ is an $n$-matrix.

Consider $j=1$. Since $M$ is not a 1 -matrix, there exists at least one $1 \times d_{2} \times d_{3} \times \cdots \times d_{n}$ submatrix that does not contain a 1 . Without loss of generality, let $M\left[i_{1},:, \ldots,:\right]$ with $1 \leqslant i_{1} \leqslant d_{1}$ be such a matrix. Next, consider $j=2$. Since $M$ is also not a 2-matrix, let $M\left[:, i_{2},:, \ldots,:\right]$ with $1 \leqslant i_{2} \leqslant d_{2}$ be a $d_{1} \times 1 \times d_{3} \times \cdots \times d_{n}$ submatrix that does not contain a 2 . Therefore, $M\left[i_{1}, i_{2},:, \ldots,:\right]$ is a $1 \times 1 \times d_{3} \times \cdots \times d_{n}$ submatrix that contains neither a 1 nor a 2 . We continue this pattern for $1 \leqslant j \leqslant n-1$. Since $M$ is not a $j$-matrix for $1 \leqslant j \leqslant n-1$, let $M\left[i_{1}, \ldots, i_{n-1},:\right]$ be the $1 \times 1 \cdots 1 \times d_{n}$ submatrix containing no elements in the set $\{1, \cdots, n-1\}$. Therefore, for all $1 \leqslant i \leqslant d_{n}, M\left[i_{1}, \ldots, i_{n-1}, i\right]=n$, all of the $d_{1} \times \cdots \times d_{n-1} \times 1$ submatrices of $M$ contain an entry with value $n$. Thus, $M$ is an $n$-matrix.

We now present the proofs of Theorems 1 through 3.

\subsection{Proof of Theorem 1}

Proof. Let $\left\{u_{1}^{1}, \ldots, u_{\gamma\left(A_{1}\right)}^{1}\right\}$ be a $\gamma$-set of $A_{1}$. Partition $V\left(A_{1}\right)$ into sets $D_{1}^{1}, \ldots, D_{\gamma\left(A_{1}\right)}^{1}$ such that $u_{j}^{1} \in D_{j}^{1} \subseteq N_{A_{1}}\left[u_{j}^{1}\right]$ for $1 \leqslant j \leqslant \gamma\left(A_{1}\right)$. Having partitioned $V\left(A_{1}\right)$ based on a minimum dominating set, we will now partition each of $V\left(A_{2}\right), \ldots, V\left(A_{n}\right)$ based on a minimum total dominating set. For $i=2, \ldots, n$, let $\left\{u_{1}^{i}, \ldots, u_{\gamma_{t}\left(A_{i}\right)}^{i}\right\}$ be a $\gamma_{t}$-set of $A_{i}$, and $D_{1}^{i}, \ldots, D_{\gamma_{t}\left(A_{i}\right)}^{i}$ be the corresponding partitions of $V\left(A_{i}\right)$ such that $D_{j}^{i} \subseteq N_{A_{i}}\left(u_{j}^{i}\right)$. Note that this implies $u_{j}^{i} \notin D_{j}^{i}$.

Now let $Q=\left\{D_{1}^{1}, \ldots, D_{\gamma\left(A_{1}\right)}^{1}\right\} \times\left\{D_{1}^{2}, \ldots, D_{\gamma_{t}\left(A_{2}\right)}^{2}\right\} \times \cdots \times\left\{D_{1}^{n}, \ldots, D_{\gamma_{t}\left(A_{n}\right)}^{n}\right\}$. Then $Q$ forms a partition of $V\left(A_{1} \square \cdots \square A_{n}\right)$ with $|Q|=\gamma\left(A_{1}\right) \prod_{i=2}^{n} \gamma_{t}\left(A_{i}\right)$.

Let $D$ be a $\gamma$-set of $A_{1} \square \cdots \square A_{n}$. Then, for each $u \in V\left(A_{1} \square \cdots \square A_{n}\right)$ not in $D$, there exists an $i$ such that $N_{\square A_{i}}(u) \cap D$ is non-empty. Based on this observation, we define an $n$-ary $\left|V\left(A_{1}\right)\right| \times \cdots \times\left|V\left(A_{n}\right)\right|$ matrix $F$ such that: 


$$
F\left(u_{1}, \ldots, u_{n}\right)= \begin{cases}1 & \text { if }\left(u_{1} \cdots u_{n}\right) \in D, \text { else } \\ i_{\text {min }} & \text { where } i_{\text {min }}=\min \left\{i \mid N_{\square A_{i}}\left(u_{1} \cdots u_{n}\right) \cap D \neq \emptyset\right\} .\end{cases}
$$

Observe that $F\left(u_{1}, \ldots, u_{n}\right)=1$ has two meanings: either $\left(u_{1} \cdots u_{n}\right) \in D$ or $\left(u_{1} \cdots u_{n}\right)$ is dominated an an $A_{1}$-edge.

For $j=1, \ldots, n$, let $d_{j} \subseteq Q$ be the set of the elements in $Q$ such that the corresponding submatrices of $F$ are $j$-matrices. By Prop. 2, each element of $Q$ belongs to at least one

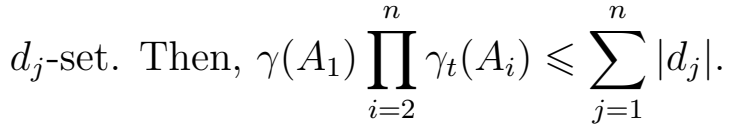

Claim 1. $\left|d_{1}\right| \leqslant|D|$.

Proof. Similar to $Q$, let $B=\left\{D_{1}^{2}, \ldots, D_{\gamma_{t}\left(A_{2}\right)}^{2}\right\} \times \cdots \times\left\{D_{1}^{n}, \ldots, D_{\gamma_{t}\left(A_{n}\right)}^{n}\right\}$. For convenience, denote $B$ as $\left\{B_{1}, \ldots, B_{|B|}\right\}$, where $|B|=\prod_{i=2}^{n} \gamma_{t}\left(A_{i}\right)$.

For $p=1, \ldots,|B|$, let $Z_{p}=D \cap\left(A_{1} \times B_{p}\right)$, and

$$
\begin{aligned}
& S_{p}=\left\{D_{x}^{1} \mid\right. \text { the submatrix of } F \text { determined by } D_{x}^{1} \times B_{p} \text { is a 1-matrix, } \\
&\text { with } \left.x \in\left\{1, \ldots, \gamma\left(A_{1}\right)\right\}\right\} .
\end{aligned}
$$

Note that if $D_{x}^{1} \times B_{p}$ is a 1 -matrix for some $x \in\left\{1, \ldots, \gamma\left(A_{1}\right)\right\}$ and $p \in\{1, \ldots,|B|\}$, then for each $w \in D_{x}^{1}$, the submatrix of $F$ corresponding to $\{w\} \times B_{p}$ contains a 1 . Therefore, $\{w\} \times B_{p}$ contains a vertex in $D$ or a vertex that is dominated by an $A_{1}$-edge. In either case, $\{w\} \times B_{p}$ contains a vertex that is dominated by $Z_{p}$. Thus, the projection of $D_{x}^{1} \times B_{p}$ on $A_{1}$ (i.e. $D_{x}^{1}$ ) is dominated by the projection of $Z_{p}$ on $A_{1}$.

We now claim that for $p=1, \ldots,|B|,\left|S_{p}\right| \leqslant\left|Z_{p}\right|$. Let $S_{p}=\left\{D_{i_{1}}^{1}, D_{i_{2}}^{1}, \ldots, D_{i_{t}}^{1}\right\}$ and let $\Phi_{A_{1}}\left(Z_{p}\right)$ be the projection of $Z_{p}$ on $A_{1}$. Then, $\Phi_{A_{1}}\left(Z_{p}\right)$ dominates $\cup_{x=1}^{t} D_{i_{x}}^{1}$, and for $i \notin\left\{i_{1}, i_{2}, \ldots, i_{t}\right\}, u_{i}^{1}$ dominates $D_{i}^{1}$. Thus, $\Phi_{A_{1}}\left(Z_{p}\right) \cup\left\{u_{i}^{1} \mid i \notin\left\{i_{1}, i_{2}, \ldots, i_{t}\right\}\right\}$ is a dominating set for $A_{1}$. Now, $\left|\Phi_{A_{1}}\left(Z_{p}\right) \cup\left\{u_{i}^{1} \mid i \notin\left\{i_{1}, i_{2}, \ldots, i_{t}\right\}\right\}\right| \geqslant \gamma\left(A_{1}\right)$, and $\mid\left\{u_{i}^{1} \mid i \notin\right.$ $\left.\left\{i_{1}, i_{2}, \ldots, i_{t}\right\}\right\} \mid=\gamma\left(A_{1}\right)-t$. Thus, $\left|\Phi_{A_{1}}\left(Z_{p}\right)\right| \geqslant t$. Hence, $\left|S_{p}\right|=t \leqslant\left|\Phi_{A_{1}}\left(Z_{p}\right)\right| \leqslant\left|Z_{p}\right|$. Finally, we see that $\left|d_{1}\right|=\sum_{p=1}^{|B|}\left|S_{p}\right| \leqslant \sum_{p=1}^{|B|}\left|Z_{p}\right|=|D|$.

Claim 2. For $j=2, \ldots, n,\left|d_{j}\right| \leqslant|D|$.

Proof. We prove here that $\left|d_{n}\right| \leqslant|D|$, but a similar proof can be performed for any other $j \geqslant 2$. Let $B=\left\{D_{1}^{1}, \ldots, D_{\gamma\left(A_{1}\right)}^{1}\right\} \times\left\{D_{1}^{2}, \ldots, D_{\gamma_{t}\left(A_{2}\right)}^{2}\right\} \times \cdots \times\left\{D_{1}^{n-1}, \ldots, D_{\gamma_{t}\left(A_{n-1}\right)}^{n-1}\right\}$. For convenience, denote $B$ as $\left\{B_{1}, \ldots, B_{|B|}\right\}$, where $|B|=\gamma\left(A_{1}\right) \prod_{i=2}^{(n-1)} \gamma_{t}\left(A_{i}\right)$.

For $p=1, \ldots,|B|$, let $Z_{p}=D \cap\left(B_{p} \times A_{n}\right)$, and

$$
\begin{aligned}
& S_{p}=\left\{D_{x}^{n} \mid\right. \text { the submatrix of } F \text { determined by } B_{p} \times D_{x}^{n} \text { is an } n \text {-matrix, } \\
&\text { with } \left.x \in\left\{1, \ldots, \gamma_{t}\left(A_{n}\right)\right\}\right\} .
\end{aligned}
$$


Note that if $B_{p} \times D_{x}^{n}$ is a $n$-matrix for some $p \in\{1, \ldots,|B|\}$ and $x \in\left\{1, \ldots, \gamma_{t}\left(A_{n}\right)\right\}$, then, for each $w \in D_{x}^{n}$, the submatrix of $F$ corresponding to $B_{p} \times\{w\}$ contains an $n$. Therefore, each $B_{p} \times\{w\}$ contains a vertex that is dominated by an $A_{n}$-edge. Therefore, each vertex in the projection of $B_{p} \times D_{x}^{n}$ on $A_{n}$ (i.e., each vertex $w \in D_{x}^{n} \subseteq V\left(A_{n}\right)$ ) is dominated by a vertex from $\Phi_{A_{n}}\left(Z_{p}\right)$, other than itself. In other words, the projection of $B_{p} \times D_{x}^{n}$ on $A_{n}$ (i.e. $D_{x}^{n}$ ) is non-self-dominated by the projection of $Z_{p}$ on $A_{n}$.

We now claim that for $p=1, \ldots,|B|,\left|S_{p}\right| \leqslant\left|Z_{p}\right|$. Let $S_{p}=\left\{D_{i_{1}}^{n}, D_{i_{2}}^{n}, \ldots, D_{i_{t}}^{n}\right\}$ and let $\Phi_{A_{n}}\left(Z_{p}\right)$ be the projection of $Z_{p}$ on $A_{n}$. Then, $\cup_{x=1}^{t} D_{i_{x}}^{n}$ is non-self-dominated by $\Phi_{A_{n}}\left(Z_{p}\right)$, and $\Phi_{A_{n}}\left(Z_{p}\right) \cup\left\{u_{i}^{n} \mid i \notin\left\{i_{1}, i_{2}, \ldots, i_{t}\right\}\right\}$ is a total dominating set of $A_{n}$. Now, $\left|\Phi_{A_{n}}\left(Z_{p}\right) \cup\left\{u_{i}^{n} \mid i \notin\left\{i_{1}, i_{2}, \ldots, i_{t}\right\}\right\}\right| \geqslant \gamma_{t}\left(A_{n}\right)$, and $\left|\left\{u_{i}^{n} \mid i \notin\left\{i_{1}, i_{2}, \ldots, i_{t}\right\}\right\}\right|=$ $\gamma_{t}\left(A_{n}\right)-t$. Thus, $\left|\Phi_{A_{n}}\left(Z_{p}\right)\right| \geqslant t$. Hence, $\left|S_{p}\right|=t \leqslant\left|\Phi_{A_{n}}\left(Z_{p}\right)\right| \leqslant\left|Z_{p}\right|$. Finally, we see that $\left|d_{n}\right|=\sum_{p=1}^{|B|}\left|S_{p}\right| \leqslant \sum_{p=1}^{|B|}\left|Z_{p}\right|=|D|$.

To conclude the proof, we observe that

$$
\gamma\left(A_{1}\right) \prod_{i=2}^{n} \gamma_{t}\left(A_{i}\right) \leqslant \sum_{j=1}^{n}\left|d_{j}\right| \leqslant n|D|=n \gamma\left(A_{1} \square \cdots \square A_{n}\right) .
$$

We conclude this section with the following corollary.

Corollary. Given graphs $G$ and $H$ containing no isolated vertices,

$$
\max \left\{\gamma(G) \gamma_{t}(H), \gamma_{t}(G) \gamma(H)\right\} \leqslant 2 \gamma(G \square H)
$$

\subsection{Proof of Theorem 2}

Proof. For $i=1, \ldots, n$, let $\left\{u_{1}^{i}, \ldots, u_{\gamma_{t}\left(A_{i}\right)}^{i}\right\}$ be a $\gamma_{t}$-set of $A_{i}$, and $D_{1}^{i}, \ldots, D_{\gamma_{t}\left(A_{i}\right)}^{i}$ be the corresponding partitions of $V\left(A_{i}\right)$ such that $D_{j}^{i} \subseteq N_{A_{i}}\left(u_{j}^{i}\right)$.

Let $Q=\left\{D_{1}^{1}, \ldots, D_{\gamma_{t}\left(A_{1}\right)}^{1}\right\} \times \cdots \times\left\{D_{1}^{n}, \ldots, D_{\gamma_{t}\left(A_{n}\right)}^{n}\right\}$. Then $Q$ forms a partition of $V\left(A_{1} \square \cdots \square A_{n}\right)$ with $|Q|=\prod_{i=1}^{n} \gamma_{t}\left(A_{i}\right)$.

Let $D$ be a $\gamma_{t}$-set of $A_{1} \square \cdots \square A_{n}$. Then, for each $u \in V\left(A_{1} \square \cdots \square A_{n}\right)$, there exists an $i$ such that $N_{\square A_{i}}(u) \cap D$ is non-empty. Based on this observation we define an $n$-ary $\left|V\left(A_{1}\right)\right| \times \cdots \times\left|V\left(A_{n}\right)\right|$ matrix $F$ such that:

$$
F\left(u_{1}, \ldots, u_{n}\right)=\min \left\{i \mid N_{\square A_{i}}\left(u_{1} \cdots u_{n}\right) \cap D \neq \emptyset\right\} .
$$

For $j=1, \ldots, n$, let $d_{j} \subseteq Q$ be the set of the elements in $Q$ which are $j$-matrices. By Prop. 2, each element of $Q$ belongs to at least one $d_{j}$-set. Then, $\prod_{i=1}^{n} \gamma_{t}\left(A_{i}\right) \leqslant \sum_{j=1}^{n}\left|d_{j}\right|$. 
Claim 3. For $j=1, \ldots, n,\left|d_{j}\right| \leqslant|D|$.

Proof. The proof is copy of proof of Claim 2, except that here $B=\left\{D_{1}^{1}, \ldots, D_{\gamma_{t}\left(A_{1}\right)}^{1}\right\} \times$ $\cdots \times\left\{D_{1}^{n-1}, \ldots, D_{\gamma_{t}\left(A_{n-1}\right)}^{n-1}\right\}$, and $|B|=\prod_{i=1}^{(n-1)} \gamma_{t}\left(A_{i}\right)$.

Thus we have, $\prod_{i=1}^{n} \gamma_{t}\left(A_{i}\right) \leqslant \sum_{j=1}^{n}\left|d_{j}\right| \leqslant n|D|=n \gamma_{t}\left(A_{1} \square \cdots \square A_{n}\right)$.

We conclude by observing that Ho's inequality follows as a corollary to Thm. 2 .

Corollary (Ho [4]). Given graphs $G$ and $H$ containing no isolated vertices,

$$
\gamma_{t}(G) \gamma_{t}(H) \leqslant 2 \gamma_{t}(G \square H) .
$$

\subsection{Proof of Theorem 3}

Proof. For $i=1, \ldots, n$, let $k_{i}=\gamma_{p r}\left(A_{i}\right) / 2$, and $\left\{x_{1}^{i}, y_{1}^{i}, \ldots, x_{k_{i}}^{i}, y_{k_{i}}^{i}\right\}$ be a $\gamma_{p r}$-set of $A_{i}$, where for each $A_{i}$ and $1 \leqslant j \leqslant k_{i},\left(x_{j}^{i}, y_{j}^{i}\right) \in E\left(A_{i}\right)$. Partition each $V\left(A_{i}\right)$ into sets $D_{1}^{i}, \ldots, D_{k_{i}}^{i}$, such that $\left\{x_{j}^{i}, y_{j}^{i}\right\} \subseteq D_{j}^{i} \subseteq N_{A_{i}}\left[x_{j}^{i}\right] \cup N_{A_{i}}\left[y_{j}^{i}\right]$ for $1 \leqslant j \leqslant k_{i}$.

Let $Q=\left\{D_{1}^{1}, \ldots, D_{k_{1}}^{1}\right\} \times \cdots \times\left\{D_{1}^{n}, \ldots, D_{k_{n}}^{n}\right\}$. Then $Q$ forms a partition of $V\left(A_{1} \square \cdots \square A_{n}\right)$ with $|Q|=\prod_{i=1}^{n} \gamma_{p r}\left(A_{i}\right) / 2=\frac{1}{2^{n}} \prod_{i=1}^{n} \gamma_{p r}\left(A_{i}\right)$.

Let $D$ be a $\gamma_{p r}$-set of $A_{1} \square \cdots \square A_{n}$. Then, for each $u \in V\left(A_{1} \square \cdots \square A_{n}\right)$, there exists an $i$ such that $N_{\square A_{i}}(u) \cap D$ is non-empty. Based on this observation, we define $n$ different matrices $F^{i}$ with $i=1, \ldots, n$, where each of the $n$ matrices is an $n$-ary $\left|V\left(A_{1}\right)\right| \times \cdots \times$ $\left|V\left(A_{n}\right)\right|$ matrix $F^{i}$ such that:

$$
F^{i}\left(u_{1}, \ldots, u_{n}\right)= \begin{cases}i & \text { if } u_{1} \cdots u_{n} \in D, \text { else } \\ j_{\min } & \text { where } j_{\min }=\min \left\{j \mid N_{\square A_{j}}\left(u_{1} \cdots u_{n}\right) \cap D \neq \emptyset\right\} .\end{cases}
$$

Thus, each of the $n$ matrices $F^{i}$ with $i=1, \ldots, n$ differs only in the entries that correspond to vertices in the paired dominating set $D$.

For $j=1, \ldots, n$ and $i=1, \ldots, n$, let $d_{j}^{i} \subseteq Q$ be the set of the elements in $Q$ which are $j$-matrices in the matrix $F^{i}$. By Prop. 2, each element of $Q$ belongs to at least one $d_{j}^{i}$-set for each $i=1, \ldots, n$. Now, if an element $q \in Q$ belongs to the $d_{j}^{i}$-set, then $q$ also belongs to the $d_{j}^{j}$-set. To see this, if $M_{i}$ and $M_{j}$ are the submatrices determined by $q$ with respect to the matrices $F^{i}$ and $F^{j}$, respectively, then all the entries that do not match in $M_{i}$ and $M_{j}$ have value $j$ in $M_{j}$. Thus, each $q \in Q$ belongs to at least one $d_{i}^{i}$-set for some $i \in\{1, \ldots, n\}$. Then, $\frac{1}{2^{n}} \prod_{i=1}^{n} \gamma_{p r}\left(A_{i}\right) \leqslant \sum_{i=1}^{n}\left|d_{i}^{i}\right|$. 
Similar to $Q$, let $B=\left\{D_{1}^{1}, \ldots, D_{k_{1}}^{1}\right\} \times \cdots \times\left\{D_{1}^{n-1}, \ldots, D_{k_{n-1}}^{n-1}\right\}$. For convenience, we denote $B$ as $\left\{B_{1}, \ldots, B_{|B|}\right\}$, where $|B|=\prod_{i=1}^{n-1} \gamma_{p r}\left(A_{i}\right) / 2=\frac{1}{2^{n-1}} \prod_{i=1}^{n-1} \gamma_{p r}\left(A_{i}\right)$. Since $D$ is a $\gamma_{p r}$-set, the subgraph of $A_{1} \square \cdots \square A_{n}$ induced by $D$ has a perfect matching. Let

$$
D_{i}=\left\{u \in D \mid \text { the matching edge incident to } u \text { is in } E_{i}\right\} .
$$

Then, $D$ can be written as the disjoint union of the subsets $D_{i}$. For $p=1, \ldots,|B|$ and $i=1, \ldots, n$, let $Z_{p}^{i}=D_{i} \cap\left(B_{p} \times A_{n}\right)$, and

$$
\begin{aligned}
S_{p}=\left\{D_{x}^{n} \mid\right. & \text { the submatrix of } F^{n} \text { determined by } B_{p} \times D_{x}^{n} \text { is an } n \text {-matrix, } \\
& \text { with } \left.x \in\left\{1, \ldots, k_{n}\right\}\right\} .
\end{aligned}
$$

Then, $\left|d_{n}^{n}\right|=\sum_{p=1}^{|B|}\left|S_{p}\right|$.

Claim 4. For $p=1, \ldots,|B|, 2\left|S_{p}\right| \leqslant 2\left|Z_{p}^{1}\right|+\cdots+2\left|Z_{p}^{n-1}\right|+\left|Z_{p}^{n}\right|$.

Proof. Let $S_{p}=\left\{D_{j_{1}}^{n}, D_{j_{2}}^{n}, \ldots, D_{j_{t}}^{n}\right\}$, and for $j=1, \ldots, n$, let $V_{j}=\Phi_{A_{n}}\left(Z_{p}^{j}\right)$. Note that $\left|V_{j}\right| \leqslant\left|Z_{p}^{j}\right|$. Additionally, let $C=\left\{x_{j}^{n} \mid j \notin\left\{j_{1}, j_{2}, \ldots, j_{t}\right\}\right\} \cup\left\{y_{j}^{n} \mid j \notin\left\{j_{1}, j_{2}, \ldots, j_{t}\right\}\right\}$.

Let $M$ be the matching on $V_{n} \cup C$ formed by taking all of the $\left\{x_{j}^{n}, y_{j}^{n}\right\}$ edges induced by the vertices in $C$, and then adding the edges from a maximal matching on the remaining unmatched vertices in $V_{n}$. Then, $E=V_{1} \cup \cdots \cup V_{n} \cup C$ is a dominating set of $A_{n}$ with $M$ as a matching. Let $M_{1}=V(M)$ and $M_{2}=\left(V_{n} \cup C\right) \backslash M_{1}$. We note that $M_{1}$ consists of all the vertices in $C$ plus the matched vertices from $V_{n}$, and $M_{2}$ contains only the unmatched vertices from $V_{n}$.

In order to obtain a perfect matching, we recursively modify $E$ by choosing an unmatched vertex $a$ in $E$, and then either matching it with an appropriate vertex, or removing it from $E$. Specifically, if $N_{A_{n}}(a) \backslash V(M)$ is non-empty, there exists a vertex $a^{\prime} \in N_{A_{n}}(a) \backslash V(M)$ such that we can add $a^{\prime}$ to $E$ and $\left(a, a^{\prime}\right)$ to the matching $M$. Otherwise, $a$ is incident on only matched vertices, and we can safely remove it from $E$ without altering the fact that $E$ is a dominating set.

Our recursively modified $E$ (denoted by $E_{\text {rec }}$ ) is now a paired dominating set of $A_{n}$. Furthermore, in the worst case, we have doubled the unmatched vertices from $V_{n}$, and also doubled the vertices in $V_{1}, \ldots, V_{n-1}$. Thus,

$$
2 k_{n} \leqslant\left|E_{\text {rec }}\right| \leqslant 2\left|V_{1}\right|+\cdots+2\left|V_{n-1}\right|+\left|M_{1}\right|+2\left|M_{2}\right| .
$$

This implies that $2 k_{n}-|C| \leqslant 2\left|V_{1}\right|+\cdots+2\left|V_{n-1}\right|+\left|Z_{p}^{n}\right|$. Since $2 k_{n}-|C|=2\left|S_{p}\right|$, therefore, $2\left|S_{p}\right| \leqslant 2\left|V_{1}\right|+\cdots+2\left|V_{n-1}\right|+\left|Z_{p}^{n}\right| \leqslant 2\left|Z_{p}^{1}\right|+\cdots+2\left|Z_{p}^{n-1}\right|+\left|Z_{p}^{n}\right|$.

Using Claim 4, we now see

$$
2 \sum_{p=1}^{|B|}\left|S_{p}\right| \leqslant \sum_{p=1}^{|B|}\left(2 \sum_{j=1}^{n}\left|Z_{p}^{j}\right|-\left|Z_{p}^{n}\right|\right)=2|D|-\sum_{p=1}^{|B|}\left|Z_{p}^{n}\right|=2|D|-\left|D_{n}\right| .
$$


Therefore, $2\left|d_{n}^{n}\right| \leqslant 2|D|-\left|D_{n}\right|$. Similarly, we can show that $2\left|d_{i}^{i}\right| \leqslant 2|D|-\left|D_{i}\right|$ for $i=1, \ldots, n$. To conclude the proof, we see

$$
\begin{gathered}
\frac{1}{2^{n-1}} \prod_{i=1}^{n} \gamma_{p r}\left(A_{i}\right)= \\
2\left(k_{1} \cdots k_{n}\right) \leqslant 2 \sum_{i=1}^{n}\left|d_{i}^{i}\right| \leqslant 2 n|D|-\sum_{i=1}^{n}\left|D_{i}\right|=(2 n-1)|D|, \\
\prod_{i=1}^{n} \gamma_{p r}\left(A_{i}\right) \leqslant 2^{n-1}(2 n-1) \gamma_{p r}\left(A_{1} \square \cdots \square A_{n}\right) .
\end{gathered}
$$

We conclude this section by observing that an improvement to the Hou-Jiang [5] inequality $\gamma_{p r}(G) \gamma_{p r}(H) \leqslant 7 \gamma_{p r}(G \square H)$ follows as a corollary:

Corollary. Given graphs $G$ and $H$ containing no isolated vertices,

$$
\gamma_{p r}(G) \gamma_{p r}(H) \leqslant 6 \gamma_{p r}(G \square H) \text {. }
$$

\section{Acknowledgments}

The authors would like to acknowledge the support of NSF-CMMI-0926618, the Rice University VIGRE program (NSF DMS-0739420 and EMSW21-VIGRE), and the Global Initiatives Fund (Brown School of Engineering at Rice University), under the aegis of SURGE (Summer Undergraduate Research Grant for Excellence), a joint program with the IIT Kanpur and the Rice Center for Engineering Leadership. Additionally, the authors acknowledge the support of NSF DSS-0729251, DSS-0240058, and the Defense Advanced Research Projects Agency under Award No. N66001-10-1-4040. Finally, the authors would like to thank the anonymous referees for their careful reading and insightful comments which greatly simplified the presentation of the proof.

\section{References}

[1] B. Brešar, P. Dorbec, W.Goddard, B.Hartnell, M.Henning, S.Klavžar, and D.Rall. Vizing's conjecture: A survey and recent results. Journal of Graph Theory, 69(1):4676, 2012.

[2] B. Brešar, M. A. Henning, and D. F. Rall. Paired-domination of Cartesian products of graphs. Utilitas Mathematica, 73:255-265, 2007.

[3] W. Clark and S. Suen. An inequality related to Vizing's conjecture. Electronic Journal of Combinatorics, 7:N4, 2000.

[4] P. T. Ho. A note on the total domination number. Utilitas Mathematica, 77:97-100, 2008.

[5] X. M. Hou and F. Jiang. Paired domination of Cartesian products of graphs. Journal of Mathematical Research \&3 Exposition, 30(1):181-185, 2010. 